\title{
Characteristics of multiple sclerosis patient stance control disorders, measured by means of posturography and related to brainstem lesions
}

\author{
Dario Alpini, ${ }^{1}$ Federica Di Berardino, ${ }^{2}$ Valentina Mattei, ${ }^{1}$ Domenico Caputo, ${ }^{1}$ \\ Peter Schalek, ${ }^{3}$ Antonio Cesarani ${ }^{2,4}$ \\ ${ }^{1}$ Scientific Institute S. Maria Nascente, Don Carlo Gnocchi Foundation, Milan, Italy; ${ }^{2}$ Dipartimento \\ di Scienze Chirurgiche Specialistiche, Università degli Studi di Milano, Milan, Italy; ${ }^{3}$ ENT Institute \\ III Medical Faculty Charles University, Prague, Czech Republik; ${ }^{4}$ Audiology Unit, Fondazione \\ IRCCS Ca' Granda Ospedale Maggiore Policlinico, Milan, Italy
}

\begin{abstract}
Balance disorders are commonly observed during the course of multiple sclerosis (MS). The aim of this study is to report characteristics of MS patient stance control disorders, measured by means of posturography and related to the brainstem lesions. Thirty-eight patients affected by MS, mildly to moderately disable according to Kurtzke's Expanded Disability Status Scale, underwent a complete clinical neurological and vestibular evaluation and brain MRI scanning. All patients were then tested on a static posturography platform (Tetrax, Israel) in four conditions: eyes open and eyes closed standing on a firm surface and on a foam pad. Clinical and/or magnetic
\end{abstract}

Correspondence: Federica Di Berardino, Audiology Unit, I.R.C.C.S. Policlinico, Mangiagalli e Regina Elena, Dipartimento di Scienze, Chirurgiche Specialistiche, University of Milan, via Pace 9 - 20122 Milano, Italy.

Tel. +39.02.5503.3922 - Fax: +39.02 .70126502 .

E-mail: federica.diberardino@unimi.it

Key words: multiple sclerosis, magnetic resonance imaging, posturography, vestibulo-spinal reflex.

Acknowledgments: the authors are grateful to Prof. Kohen-Raz of the Hebrew University, Jerusalem, Israel, for useful comments and to Stella Forti for her technical support.

Data has been shown by D. Alpini, D. Caputo, D. Pellegatta and M. Fini at the Proceedings of the $10^{\text {th }}$ Meeting of the European Neurological Society, Jerusalem, Isreal, 2000.

Contributions: DA and FDB wrote the article; VM and DC performed tests; PS organized data collection and performed data analysis; AC supervised and reviewed the article.

Conflicts of interest: the authors declare no potential conflicts of interest.

Received for publication: 30 September 2011.

Revision received: 30 December 2011.

Accepted for publication: 3 January 2012.

This work is licensed under a Creative Commons Attribution NonCommercial 3.0 License (CC BY-NC 3.0).

@C Copyright D. Alpini et al., 2012

Licensee PAGEPress, Italy

Audiology Research 2012; 2:e9

doi:10.4081/audiores.2012.e9 resonance imaging evidence of brainstem involvement was observed in $55.3 \%$ of patients. When brainstem lesion was detected, Fourier analysis showed a typical pattern characterized by inversion of the 0 $0.1 \mathrm{~Hz}$ and $0.1-0.25 \mathrm{~Hz}$ frequency bands. In conclusion, MS leads to pervasive postural disturbances in the majority of subjects, including the visuo-vestibular loops and proprioception involving vestibulospinal pathways in at least $55.3 \%$ of patients. Our results may also suggest the presence of Fourier inversion in patients with brainstem lesions.

\section{Introduction}

Balance disorders are commonly observed during the course of multiple sclerosis (MS). Though disequilibrium mostly arises from an involvement of the vestibulo-spinal system in these subjects, the visual-vestibulo-oculomotor reflexes remain the most often investigated. ${ }^{1}$

MS can affect all areas of the central nervous system and cause such a wide range of impairments that control changes in people with MS may be thought to have multifactorial causes. A review by Cameron $e t$ $a l^{2}$ showed that changes in postural control in patients with MS are most likely to be the result of slowed somatosensory conduction and impaired central integration control. In fact, it is well known that brainstem and cerebellum involvement is likely in many MS patients and that it is severely and rapidly affected by this pathology.

More recently, perspectives of surgical treatment have been proposed in some cases of MS. ${ }^{3}$ Functional evaluations in the follow up of MS have, therefore, become the focus of greater importance since follow-up gold standards are still to be defined. ${ }^{4}$ Among the functional evaluations considered, static posturography has often been used to study sensory impairments during quiet standing in subjects with MS..$^{5-9}$ Static posturography measures displacement of the center of pressure on a force-measuring platform and allows precise evaluation of the postural strategies employed by patients suffering from a variety of vestibular disorders. ${ }^{8,10}$ Its major limitation is the patient's ability to maintain a sufficiently stable stance for the duration of the test (approx. $2 \mathrm{~min}$ ). Documentation of disturbances in controlling an upright position has been proposed to complete neuro-physiological assessment of MS and provide useful functional information in the planning of rehabilitative treatment. ${ }^{11-13}$ Center of pressure displacement during standing tasks has been shown to be a useful performance-based evaluation parameter also for MS patients with minimal or no clinically evident balance deficits who are not yet experiencing 
functional limitations or disability. ${ }^{14}$ On the other hand, Frzovic et al. ${ }^{15}$ reported that there were no differences between MS and control groups regarding their ability to maintain standing balance with the feet apart, together, or in stride stance. However, they did find that MS patient performance was poorer than controls in tandem and single leg stance in the functional reach test, arm raise test, step test, and in response to an external perturbation.

Cattaneo and colleagues ${ }^{16}$ recently reported a study of sensory impairment in MS patients obtained by adapting the Shumway-Cook protocol using a stabilometric platform. In this study, they underlined that previous reports on stance disturbance in MS using posturography often had not considered the importance of analyzing conditions and variables other than anteroposterior sway alone.

A recent study has shown that even mildly impaired MS patients might present measurable equilibrium disturbances upon static posturography, leading to difficulties in standing up and increasing the risk of falls. ${ }^{17}$ If so, posturography data should correlate with brainstem neuroimaging lesions and equilibrium disturbances should be identifiable at an early stage by static posturography with an interactive balance system which allows a Fourier spectral analysis. Fourier spectral analysis allows us to better identify subgroups of unsteady patients. ${ }^{18,19}$ Indeed, excessive postural sway upon Fourier spectral analysis in each of the frequency ranges reflects intensified activity within the relevant postural subsystem, either due to pathology or to compensatory efforts, ${ }^{20}$ and a variation in the low-medium sway (0.10-0.50 Hz; F2-F4) frequencies are usually considered to be an expression of vestibular control. ${ }^{21}$ This hypothesis is supported by a recent report suggesting that damage to specific infratentorial areas negatively affects static standing balance and may predispose MS patients to accidental falls. ${ }^{22}$ The aim of this study is to evaluate whether stance disorders measured by static posturography may be related to clinical signs of brainstem involvement and/or magnetic resonance imaging (MRI) brainstem lesions in MS patients with mild-moderate impairment. If so, it may be useful to include static posturography among the gold standard parameters for post-surgical MS follow up.

\section{Materials and Methods}

Posturography was recorded in 38 inpatients (22 females and 16 males) affected by primary progressive MS, clinically defined according to the Poser criteria, ${ }^{23}$ and in 32 normal subjects (18 females and 14 males, mean age $37 \pm 4.8$ years) as controls. The control group did not report any static or dynamic postural control disturbance and did not present any history of neurological or otological disease that could interfere with postural assessment.

Mean age of the patient group was $37.09 \pm 10.6$ years (range $22-58$ years), with a mean disease duration of $10.54 \pm 7.7$ years (range 0.7-31.8 years). All of the patients were mildly to moderately disable according to Kurtzke's Expanded Disability Status Scale (EDDS) ${ }^{24}$ with a median EDSS score of 2.8 (range 1.0-6.0). The presence of any ankle, knee or hip disorders that could interfere with the results was ruled out in all subjects before testing. Furthermore, other previous diseases that could interfere with balance performance, such as whiplash, head trauma or Ménière's disease, or assumption of drugs such as benzodiazepine, were excluded. All patients underwent a complete clinical neurological and vestibular evaluation and brain MRI scanning. They fulfilled the criteria of definite MS based on clinical findings, abnormal cerebrospinal fluid findings, and abnormalities on MRI of the brain and spinal cord. All patients were able to maintain autonomous stance for at least $3 \mathrm{~min}$.

Posturography with interactive balance system was recorded by means of equipment with four independent force plates (Tetrax-IBS equipment by Sunlight Medical Ltd., Tel Aviv, Israel) ${ }^{25-27}$ (CE patent $n$. 93741CE02; FDA establishment registration n. 3006701790).

Subjects were requested to stand quietly on the platforms in a quiet room in four different conditions: eyes open and eyes closed on a solid surface and eyes open and eyes closed on a foam pad in order to reduce somatosensorial and visual information.

The Tetrax posturography system used in the study consists of four independent mobile force plates, two for each foot. The plates are positioned in order to measure any equilibrium disturbance from the two forefeet and two heels. Each plate contains a strain-gauge force transducer, which is sensitive to vertical force. Tetrax provides independent output from the four balance plates and, by taking the average of all four measurements, provides a description of body sway in terms of displacement of the patient's center of pressure.

The system measures the changes and fluctuations of the vertical force exerted by the corresponding heel or forefoot during the default 30 -second experimental time and emits four separate wave signals at a sampling rate of $34 \mathrm{~Hz}$. On the basis of these fluctuations, the system computes a general stability score (ST), normalized for body weight according to the following equation:

$$
\mathrm{ST}=t\left\{\frac{\sum_{1}^{n}\left[\left(a_{n}-a_{n-1}\right)^{2}+\left(b_{n}-b_{n-1}\right)^{2}+\left(c_{n}-c_{n-1}\right)^{2}+\left(d_{n}-d_{n-1}\right)^{2}\right]}{N}\right\}^{1 / 2} / W .
$$

where $\mathrm{a}, \mathrm{b}, \mathrm{c}, \mathrm{d}$ are the four pressure transducers, $\mathrm{W}$ is body weight, $\mathrm{t}$ is experimental time, and $\mathrm{n}$ is the number of signals sampled at $34 \mathrm{~Hz}$.

\section{Quantitative sway analysis}

General stability (ST) is, therefore, calculated as an index, which refers to a mean of oscillations, recorded by each plate: the higher the value, the lower the stability. It is the quotient of the sum of the amplitudinal changes (body sway), recorded in microvolts, divided by body weight. While it is not a direct measurement of postural stability, the fluctuations of the vertical ground reaction force normalized for body weight have been found to correlate with both the static and dynamic parts of Berg's balance test and with the antero-posterior center of pressure (COP) velocity. ${ }^{28}$ In fact, the ST score provides information about the adequacy of postural adjustments. A lower ST indicates smaller fluctuations and therefore better postural control, and it has also been seen to be significantly correlated to the equilibrium score of the Equitest posturography system. ${ }^{29}$

The weight distribution index (WDI) is calculated on the basis of the relative recorded weight at each of the four supports: the higher the WDI, the higher the abnormal distribution of the weight that had to be theoretically equally divided between the four supports.

\section{Qualitative sway analysis}

Fourier analysis of sway frequencies is a mathematical treatment of wave signals of body oscillations on the horizontal plane produced by the patient in order to maintain an upright position.

The Tetrax system combines the signals from the left and right leg to result in one sway frequency with a sampling rate of $34 \mathrm{~Hz}$, as described by Kohen-Raz. ${ }^{30}$ The four signals recorded at the four sensors are analyzed with the Fourier transformation and then Tetrax recalculates an average of the results obtained providing a unique parameter. Fourier analysis is then carried out to determine which signal frequencies are most common. Fourier analysis of sway frequency distribution shows that a normal postural performance is characterized by a high intensity at the low range, and this appears to indicate that posture is controlled with minimal effort, not involving any activity of the semicircular canals, which are considered to be insensitive to body displacements below $0.2 \mathrm{~Hz} .{ }^{31}$ Fourier analysis of sway frequencies is divided into eight frequency bands (F1-F8). 
Posturography with an interactive balance system was recorded by means of equipment with four sensors; therefore, Fourier transformations were derived from four independent wave signals and were presented in the form of a spectrum, broken down into the following eight frequency bands: F1 0.01-0.1; F2 0.1-0.25; F3 0.25-0.35; F4 0.350.50 ; F5 0.50-0.75; F6 0.75-1.00; F7 1.00-3.00 Hz; and F8 above $3.0 \mathrm{~Hz}$. Previous studies on the Fourier spectral analysis of postural sway by Tetrax ${ }^{25,26,30-33}$ have shown that typical ranges of postural frequency (i.e. frequency bands) express the different levels of activity of postural subsystems, the systems that affect postural sway. Study of postural frequency can provide insights into the individual's use of these postural subsystems, which include the vestibular, somatosensory, and other sub-systems, to maintain postural stability. Thus, spectral analysis of postural sway might be a valuable tool in clinical diagnosis. Tetrax posturography has been shown to have a high test-retest reliability. ${ }^{30}$

The MRI protocol included two interleaved sets of 16 slices each covering the whole brain, which were obtained at 1.5 T (Intera, Philips Medical Systems, the Netherlands), sampling the brain at a total of 32 contiguous levels. Each of the two sets included conventional spin-echo sequences providing T1w (520/15 ms TR/TE) and PD/T2w (1800/15-90 ms TR/TE) $4 \mathrm{~mm}$ thick axial images ( $24 \mathrm{~cm}$ FOV, $256 \times 256$ acquisition matrix). ${ }^{34,35}$ Patients with acute or subacute lesions were excluded. For the purpose of the present study, only patients who presented at least two T2-weighted MRI lesions at brainstem level were considered to have imaging signs of brainstem lesion.

\section{Statistical analysis}

A descriptive statistical analysis was performed. Kurtosis/Skewness index was not significant. A Student's t-test was applied to compare posturographic findings (including Fourier analysis) in normal subjects and MS patients. Pearson's correlation was used to evaluate the possible relationships between general stability or weight distribution index and clinical signs of brainstem involvement and/or MRI brainstem lesions.

\section{Results}

Stability was normal in only 7 patients (18.4\%). In fact, the MS stability index was higher than in normal subjects in all analyzed conditions $(\mathrm{P}<0.0001)$ (Table 1). Stability in MS patients was lower with the eyes closed standing on foam pads.

Weight distribution index results are shown in Table 1 and were normal in $42 \%$. Weight distribution was slightly abnormal in MS sufferers only in tests with the eyes closed $(\mathrm{P}=\mathrm{ns})$.

Normative data of Fourier analysis are shown in Figure 1. Fourier analysis was normal in 17 patients (44.7\%). In the other patients, an atypical pattern characterized by an inversion of the frequency bands ( $F 2=0.1-0.25 \mathrm{~Hz}$ higher than $\mathrm{F} 1=0-0.1 \mathrm{~Hz}$ ) was observed (Figure 1) $(\mathrm{P}<0.05)$. In patients with clinical signs of brainstem involvement and/or MRI brainstem lesions, body sways on the horizontal plane produced in order to maintain an upright position were set on a higher frequency then in normal subjects.

Clinical and/or MRI evidence of brainstem involvement was observed in 21 of 38 (55.3\%) of patients. No relationship was found between general stability or weight distribution index and clinical signs of brainstem involvement and/or MRI brainstem lesions. On the contrary, all the subjects presenting clinical and/or imaging signs of brainstem lesion (21 of 38) had an inverted frequency distribution pattern.

\section{Discussion}

Many studies have shown that MS patients have a reduced stability in comparison with normal subjects, even in the simplest conditions (eyes open), ${ }^{15,36}$ and that instability could be primarily interpreted as the involvement of the central multisensorial control of vestibulo-spinal reflexes. ${ }^{37}$ In MS patients, balance is impaired and this impairment is greater in progressive MS than in relapsing remitting forms..$^{38}$ Gait and balance impairment can be detected in the early stages of the disease, even in the absence of clinical signs of pyramidal dysfunction. ${ }^{39}$ Therefore, the aim of this study was to relate the results of posturographic assessment to the clinical or imaging evaluation of brainstem lesions in patients with mild to moderate MS.

Our data from the quantitative analysis agree with literature; in particular, in MS patients, ST was significantly higher than in normal subjects in all conditions analyzed. On the other hand, no relationship was found between GS or WDI and clinical signs of brainstem involvement and/or MRI brainstem lesions. As expected, Fourier spectral analysis illustrated the sensory condition better than indexes of sway analysis, such as ST and WDI. In fact, ST showed only that MS gives a stability deficit that worsens in response to more difficult conditions of evaluation (visual and somatosensory deprivation), i.e. conditions that stress the vestibular control of posture and stability. This finding confirms data reported by Cattaneo and colleagues. ${ }^{16}$

Fourier spectral analysis of postural sway has been applied in several studies since the 1970 s, using different posturographic methods. ${ }^{14,32,33}$ These studies demonstrate that variations in intensity at the low, medium or high frequency ranges of the Fourier spectrum may reflect disturbances, compensatory mobilization, or functional breakdown of the visual, somatosensory and vestibular subsystems of postural control. Consequently, spectral analysis of postural sway has been

Table 1. General stability and weight distribution index in normal control subjects and in multiple sclerosis patients.

\begin{tabular}{|c|c|c|c|c|}
\hline & \multicolumn{2}{|c|}{$\begin{array}{l}\text { General } \\
\text { stability }\end{array}$} & \multicolumn{2}{|c|}{$\begin{array}{l}\text { Weight distribution } \\
\text { index }\end{array}$} \\
\hline & Control & MS & Control & MS \\
\hline Eyes open & $11.7 \pm 4.7$ & $20.7 \pm 7.8$ & $5.1 \pm 2.9$ & $6.0 \pm 2.8$ \\
\hline Eyes closed & $15.1 \pm 4.8$ & $29.9 \pm 10.1$ & $4.9 \pm 2.4$ & $7.4 \pm 3.2$ \\
\hline Eyes open+foam & $14.4 \pm 4.1$ & $29.9 \pm 10.1$ & $7.2 \pm 3.2$ & $7.6 \pm 3.4$ \\
\hline Eyes closed+foam & $21.9 \pm 8.2$ & $41.7 \pm 13.7$ & $6.6 \pm 3.3$ & $9.0 \pm 3.8$ \\
\hline
\end{tabular}

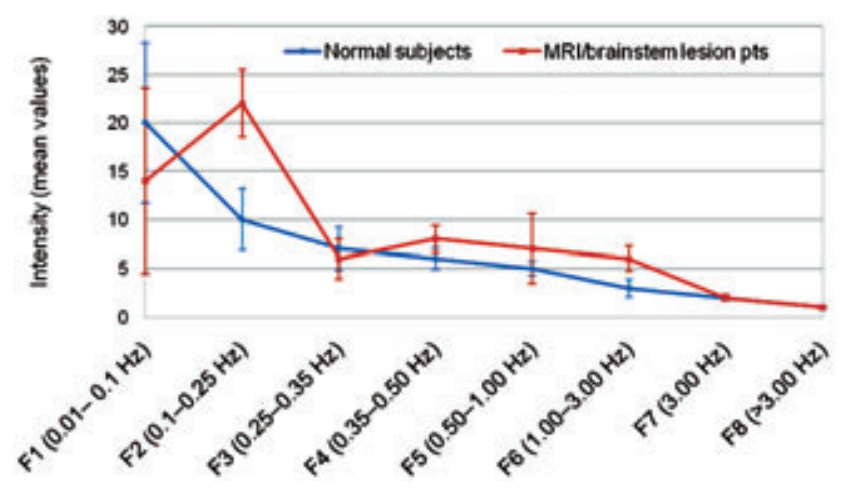

Figure 1. Fourier analysis: sway frequencies in normal subjects and in patients with clinical signs of brainstem involvement and/or magnetic resonance imaging brainstem lesions. 
proposed to be a more sensitive tool rather than unsteadiness and sway in differential clinical diagnosis. ${ }^{13}$ In particular, a low frequency range appears to indicate that posture is controlled by an intact oculomotor vestibular-otolythic mechanism. Gagey and Toupet ${ }^{40}$ identified a dominant sway at $0.2 \mathrm{~Hz}$ that corresponds to the rhythm of normal breathing, and named the multisensorial system involved in postural control at low frequencies the Fine Tuned Postural System. According to these authors, this kind of system is a functional rather than an anatomical system, involving the vestibular nuclei and posterior cerebellum that are the end points of the primary vestibular afferents. Vestibular nuclei communicate bilaterally through a commissural system that is predominantly inhibitory. Secondary vestibular neurons also receive convergent sensory information from the optokinetic circuitry system, the central visual system and the proprioceptive systems of the neck, but they cannot distinguish between sources of afferent activity. However, the firing of secondary vestibular neurons can distinguish between active and passive movements. The posterior cerebellum has extensive afferent and efferent connections with the vestibular nuclei. Primary vestibular afferents are distributed to the ipsilateral uvula-nodulus as mossy fibers whereas secondary vestibular afferents are distributed bilaterally. Climbing fibers to the cerebellum originate from two subnuclei of the contralateral inferior olive; the dorsomedial cell column and the beta-nucleus. Vestibular climbing fibers carry information only from the vertical semicircular canals and otoliths. They establish a coordinate map, set out in sagittal zones on the surface of the uvulanodulus. Purkinje cells respond to vestibular stimulation with antiphasic modulation of climbing fiber responses. The vestibulo-cerebellum imposes a vestibular coordinate system on postural responses and permits adaptive guidance of movement.

F1/F2 inversion might be a dysfunction of the Fine Tuned Postural System involving a disturbance in the visual/vestibular loops. The vestibular system appears to play an important role in this buffer mechanism. Results may suggest the presence of an MS-specific reorganization of the postural balance control system. Evidently, MS leads to pervasive postural disturbances, including visuo-vestibular loops and proprioception. Fourier abnormalities correlated to clinical and imaging signs indicate that $55.3 \%$ of MS patient instability could be due to brainstem involvement.

\section{References}

1. Alpini D, Caputo D, Pugnetti L, Giuliano DA, Cesarani A. Vertigo and multiple sclerosis: aspects of differential diagnosis. Neurol Sci 2001;22:S84-7.

2. Cameron MH, Lord S. Postural control in multiple sclerosis: implications for fall prevention. Curr Neurol Neurosci Rep 2010;10:40712.

3. Mandato KD, Hegener PF, Siskin GP, Haskal ZJ, Englander MJ, Garla S, et al. Safety of endovascular treatment of chronic cerebrospinal venous insufficiency: a report of 240 patients with multiple sclerosis. J Vasc Interv Radiol 2011. [Epub ahead of print]

4. Zivadinov R, Ramanathan M, Dolic K, Marr K, Karmon Y, Siddiqui $\mathrm{AH}$, et al. Chronic cerebrospinal venous insufficiency in multiple sclerosis: diagnostic, pathogenetic, clinical and treatment perspectives. Expert Rev Neurother 2011;11:1277-94.

5. Alpini D, Caputo D, Pellegatta D, Fini M, Kohen-Raz R. The evaluation of balance disorders in multiple sclerosis by means of tetraataxiometry. J Neurol 2000;247:136.

6. Daley M, Swank RL. Changes in postural control and vision induced by multiple sclerosis. Agressologie 1983;24:327-9.

7. Jackson R, Epstein CM, De l'Aune WR. Abnormalities in posturography and estimations of visual vertical and horizontal in multiple sclerosis. Am J Otol 1995;16:88-93.

8. Nelson SR, Di Fabio RP, Anderson JH. Vestibular and sensory interaction deficits assessed by dynamic platform posturography in patients with multiple sclerosis. Ann Otol Rhinol Laryngol 1995; 104:62-8.

9. Ramdharry GM, Marsden JF, Day BL, Thompson AJ. De-stabilizing and training effects of foot orthoses in multiple sclerosis. Mult Scler 2006;12:219-26.

10. Baloh RW, Jacobson KM, Beykirch K, Honrubia V. Static and dynamic posturography in patients with vestibular and cerebellar lesions. Arch Neurol 1998;55:649-54.

11. Alpini D, Pugnetti L, Caputo D, Cornelio F, Capobianco S, Cesarani A. Vestibular evoked myogenic potentials in multiple sclerosis: clinical and imaging correlations. Mult Scler 2004;10:316-21.

12. Crenshaw SJ, Royer TD, Richards JG, Hudson DJ. Gait variability in people with multiple sclerosis. Mult Scler 2006;12:613-9.

13. Hebert JR, Corboy JR, Manago MM, Schenkman M. Effects of vestibular rehabilitation on multiple sclerosis-related fatigue and upright postural control: a randomized controlled trial. Phys Ther 2011;91:1166-83.

14. Karst GM, Venema DM, Roehrs TG, Tyler AE. Center of pressure measures during standing tasks in minimally impaired persons with multiple sclerosis. J Neurol Phys Ther 2005;29:170-80.

15. Frzovic D, Morris ME, Vowels L. Clinical tests of standing balance: performance of persons with multiple sclerosis Arch Phys Med Rehabil 2000;81:215-21.

16. Cattaneo D, Jonsdottir J. Sensory impairments in quiet standing in subjects with multiple sclerosis. Mult Scler 2009;15:59-67.

17. Findling 0 , Sellner J, Meier N, et al. Trunk sway in mildly disabled multiple sclerosis patients with and without balance impairment. Exp Brain Res 2011;213:363-70.

18. Morad Y, Azaria B, Avni I, Barkana Y, Zadok D, Kohen-Raz R, et al. Posturography as an indicator of fatigue due to sleep deprivation. Aviat Space Environ Med 2007;78:859-63.

19. Goto F, Kabeya M, Kushiro K, et al. Effect of anxiety on antero-posterior postural stability in patients with dizziness. Neurosci Lett 2011;487:204-6.

20. Nashner LM. Organization and programming of motor activity during posture control. Prog Brain Res 1979;50:177-84.

21. Oppenheim U, Kohen-Raz R, Alex D, Kohen-Raz A, Azarya M. Postural characteristics of diabetic neuropathy. Diabetes Care 1999;22:328-32.

22. Prosperini L, Kouleridou A, Petsas N, Leonardi L, Tona F, Pantano $\mathrm{P}$, et al. The relationship between infratentorial lesions, balance deficit and accidental falls in multiple sclerosis. J Neurol Sci 2011;304:55-60.

23. Poser CM, Brinar VV. Diagnostic criteria for multiple sclerosis. Clin Neurol Neurosurg 2001;103:1-11.

24. Kurtzke JF. Rating neurologic impairment in multiple sclerosis: an expanded disability status scale (EDSS). Neurology 1983;33:144452.

25. Kohen-Raz R, Hiriartborde E. Some observations on tetra-ataxiametric patterns of static balance and their relation to mental and scholastic achievements. Percept Mot Skills 1979;48:871-90.

26. Kohen-Raz R, Volkmar FR, Cohen DJ. Postural control in children with autism. J Autism Dev Disord 1992;22:419-32.

27. Gstöttner M, Neher A, Scholtz A, Millonig M, Lembert S, Raschner C. Balance ability and muscle response of the preferred and nonpreferred leg in soccer players. Motor Control 2009;13:218-31.

28. Karlsson A, Frykberg G. Correlations between force plate measures for assessment of balance. Clin Biomech 2000;15:365-9.

29. Turner DM. Evaluation of the Tetrax Interactive Balance System and Equitest Using Normal Subjects. Degree Diss., University of Southampton, UK, 1998. 
30. Kohen-Raz R. Application of tetra-ataxiametric posturography in clinical and developmental diagnosis. Percept Mot Skills 1991;73:635-56.

31. Kohen-Raz R, Himmelfarb M, Tzur S, Kohen-Raz A, Shub Y. An initial evaluation of work fatigue and circadian changes as assessed by multiplate posturography. Percept Mot Skills 199;82:547-57.

32. De Wit G. Optic versus vestibular and proprioceptive impulses, measured by posturometry. Agressologie 1972;13:75-9.

33. Taguchi K. Spectral analysis of the movement of the center of gravity in vertiginous and ataxic patients. Agressologie 1978;19:69-70.

34. Brex PA, Ciccarelli 0, O'Riordan JI, Sailer M, Thompson AJ, Miller DH. A longitudinal study of abnormalities on MRI and disability from multiple sclerosis. N Engl J Med 2002;346:158-64.

35. Prinster A, Quarantelli M, Lanzillo R, Orefice G, Vacca G, Carotenuto B, et al. A voxel-based morphometry study of disease severity correlates in relapsing - remitting multiple sclerosis. Mult
Scler 2010;16:45-54.

36. Diener HC, Dichgans J, Bacher M, Gompf B. Quantification of postural sway in normals and patients with cerebellar diseases. Electroencephalogr Clin Neurophysiol 1984;57:134-42.

37. Ferdjallah M, Harris GF, Wertsch JJ. Instantaneous postural stability characterization using time-frequency analysis. Gait Posture 1999;10:129-34.

38. Soyuer F, Mirza M, Erkorkmaz U. Balance performance in three forms of multiple sclerosis. Neurol Res 2006;28:555-62.

39. Martin CL, Phillips BA, Kilpatrick TJ, Butzkueven H, Tubridy N, McDonald E, et al. Gait and balance impairment in early multiple sclerosis in the absence of clinical disability. Mult Scler 2006;12:620-8.

40. Gagey PM. L'amplitude des oscillations posturales dans la bande de frequence 0.2 Hertz. Etude chez le sujet normal. Paris, France: Publications de l'Institut De Posturologie;1998. 